\title{
CONTROL OF HYBRID ELECTRIC-HYDRAULIC DRIVE FOR VERTICAL FEED AXES OF MACHINE TOOLS
}

\author{
Š. Fiala ${ }^{1 *}$, A. Bubák ${ }^{1}$, L. Novotný1 \\ ${ }^{1}$ Czech Technical University in Prague, Research Center of Manufacturing Technology, Prague, Czech Republic \\ ${ }^{*}$ Corresponding author; e-mail: S.Fiala@rcmt.cvut.cz
}

\begin{abstract}
This paper deals with methods to reduce the influence of gravitational force on machine tool vertical feed axes. It provides an overview of existing counterbalancing mechanisms that are used in industrial applications. The main focus is on an existing solution that uses a rotary piston hydromotor, which has a number of advantageous properties. However, its significant torque ripple limits the practical application in machine tools. This paper presents a method of connected servo-electric and servo-hydraulic drive control that overcomes the shortcomings of the original solutions and allows practical deployment on vertical feed axes.
\end{abstract}

\section{Keywords:}

Machine tool; Vertical moving components; Counterbalancing mechanism

\section{INTRODUCTION}

Machine tool drives are usually dimensioned to meet acceleration, process and passive resistance force requirements. The most important forces for the drive design process are often acceleration forces.

Most machine tools are equipped with at least one vertical feed axis, where gravitational force also needs to be taken into account. This means that for vertical axes that are not counterbalanced the servo drive has to permanently overcome gravitational force in addition to acceleration in an upwards motion or to add force in the opposite direction when decelerating movement in a downwards motion.

The design of non-counterbalanced vertical feed drives requires special attention. It is necessary to keep in mind that there is a constant amount of current through the servo drive, which is holding the required position. Very often the servo drive needs to be larger than movement dynamics require, thus an adequate enlarged frequency converter is needed. The electrical energy consumption is also considerable. Furthermore, a larger servo drive produces more heat, which then negatively influences the thermal behavior of the machine tool.

From the aforementioned statements, it is evident that the reduction of force needed to hold vertical moving components is important. Machine tool producers have many approaches to reducing gravitational demands on servo drives. From Newton's second law it is obvious that mass impacts the resultant acceleration force. It is obvious that the gravitational force has to be reduced. Large machine tool producers address this issue by using unconventional materials, topological optimization and unusual kinematic structures or by using counterbalancing mechanisms.

\section{SOLUTIONS TO REDUCE THE LOAD ON VERTICAL AXIS DRIVES BY GRAVITY}

Research into materials for machine tool design is still in progress and there are many examples of how the structure can be improved through use of unconventional materials. In many cases experiments follow requirements on structural damping, static stiffness, thermal stability, etc. This does not necessarily mean that the weight of the component will be reduced. Here the main focus will be on methods which lead to weight reduction of components through use of unconventional materials, optimization of structure and kinematics and counterbalancing mechanisms.

To reduce the load on a drive, moving components can be designed with lighter materials. Möhring mentioned a significant reduction of mass $(60 \%)$ achieved through substitution of a conventional cast iron structure with carbon fiber reinforced composite (CFRC) by Abele at PTW Darmstadt [Möhring, H.-C. 2015]. MAP, a machine tool component producer, presented a machine tool slide made completely out of CFRC at EMO Hannover 2013 [Novotný, L. 2014]. Munirathnam experimented with metallic materials. Machine slides made as aluminum structures, welded steel structures and aluminum foam structures were tested with regard to mass, dynamic and static stiffness [Munirathnam, M. 2008]. Topological optimization in the early machine tool design phase leads to distribution of mass in order to reach the highest dynamic and static parameters with minimum material consumption. The entire process was described by Kolar et al [Kolár, P. 2017]. The bionical approach of structural optimization leads to mass reduction (3\%) and an increase in specific stiffness (24\%) [Zhao, L. 2011] with respect to human body constitution (e.g. ribs). Lightweight components like portals, slides and headstocks can be designed with identical or better 
stiffness parameters than conventional versions. Loxin uses a combination of serial and parallel kinematics on its portal PKM Tricept machine tool for large aircraft workpieces [Loxin Industrial Applications n.d.]. The manufacturer Icon Technologies has developed a standalone 6 -axis machine tool with a spindle speed of 18,000 RPM, performance of $35 \mathrm{kw}$ and a rotary table size of 800mm [ICON Technologies n.d.].

Mentioned solutions to lightweight the whole components were presented to make enumeration complete. The main focus will be paid to mechanisms intended for counterbalancing. In general, this chapter may be divided into sections in relation to the stroke-dependent character of force (see Fig. 1):

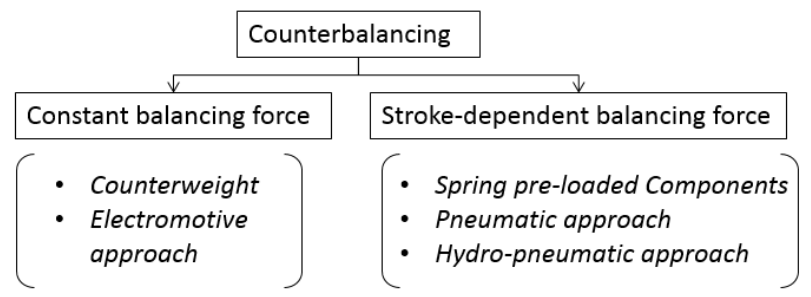

Fig. 1: Counterbalancing approaches.

Counterbalancing methods may also be classified with respect to the energy delivering intermediary.

- Mechanical Approach

- Electrical Approach

- Fluid Approach

\subsection{Mechanical Approach}

The pure mechanical counterbalancing approach is based on spillover of potential energy between specific components. The most widespread method is the use of counterweights (a very popular solution for very large machine tools). A pulley is mounted on the top of the column to reorient and transfer force held by a steel cable, which connects both moving components - i.e. headstock and counterweight (Fig. 2).

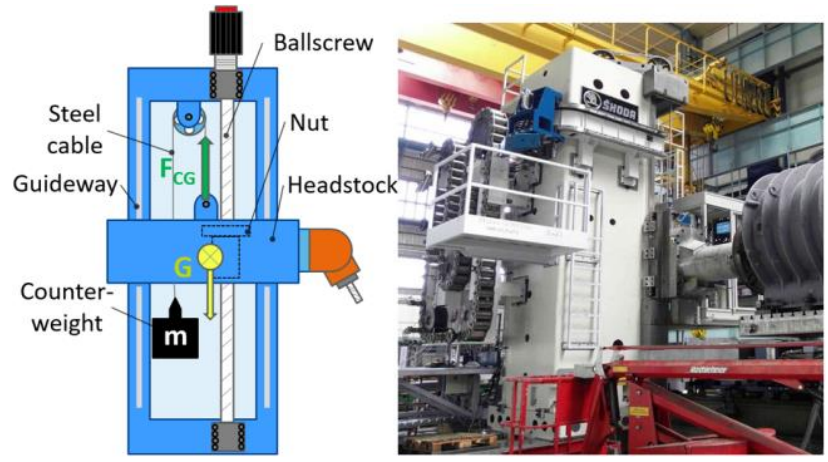

Fig. 2: Counterweight balancing method [ŠKODA MACHINE TOOL n.d.].

This solution has many advantages such as low implementation costs, simple mechanics and possible $100 \%$ compensation of gravitational force [Hovorka, J. 2015]; nevertheless there are also some disadvantages. The acceleration in an upwards motion should never reach $1 \mathrm{~g}$ because the steel cable will relax and the whole superstructure will receive impact from the re-stretched cable. There is another disadvantage associated with rope: dynamic behavior. The counterweight may be imagined as a mass on a spring connected to the headstock. This causes excitation of vibrations and thus at the very least degrades the quality of the machining process. Moreover the whole column has much more weight, which can, in the case of a movable column, cause problems on the horizontal drive. The issue associated with the counterweight and steel cable or chain is described in detail by Lin [Lin, S. 2008].

Another element, which is able to hold some potential energy, is a spring (Fig. 3). This kind of counterbalance is typical of low-cost CNC machines and in the industrial environment this solution is seen very rarely and only on smaller machines.

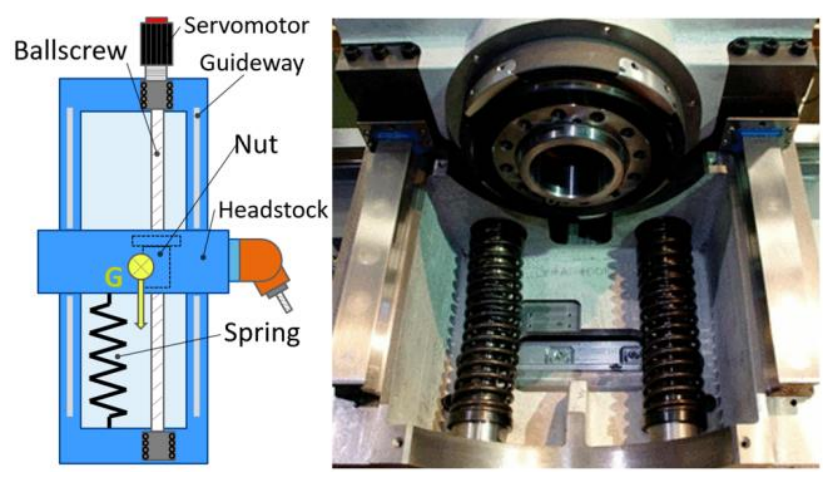

Fig. 3: Energy for counterbalancing stored in springs [Emco Group n.d.].

\subsection{Electrical Approach}

Machine tools without a special counterbalancing system may be included in this chapter because designers of such machines have to consider gravity during the servo sizing phase. The horizontally moving axis reaches the required position and control deviation of the position loop drops to zero as well as control deviation of the velocity loop. If no passive resistance or other distortion forces are considered, then the current drops to zero as well.
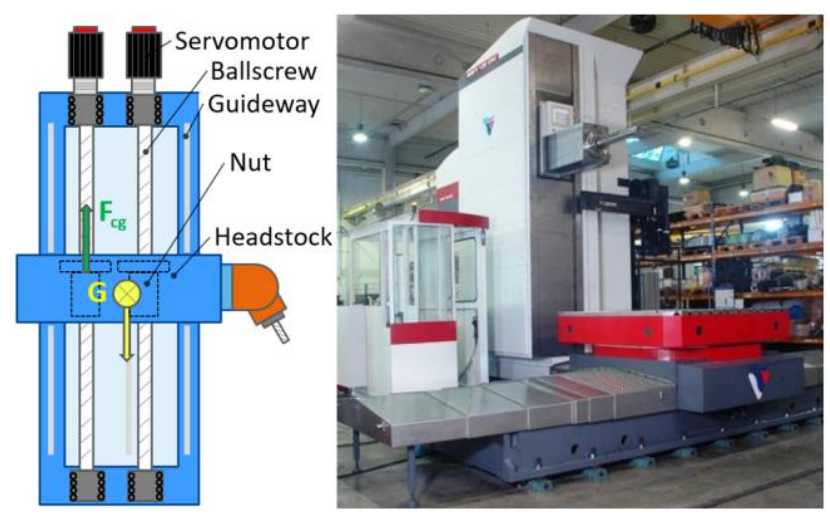

Fig. 4: Counterbalancing using identical servo drive assembly [Fermat machinery n.d.].

In the vertical case both deviations (position and velocity) drop to zero, but the actual value of the current corresponds to the gravitational force on the moving component. This current is consumed whenever the machine is on and the axis holds the position until the brake is enabled (stand-by mode, or drive disabled mode). Some machine tool producers add one identical servo drive assembly (secondary unit) to compensate the gravitational forces through force loop operation (Fig. 4) [Hovorka, J. 2015]. Adding another servo drive assembly introduces the need for an additional option for the added secondary drive. This solution does not reflect the need to reduce current consumption. 


\subsection{Fluid Approach}

In this case, fluid counterbalancing which uses compressed air as a medium and hydraulic mechanisms which use oil may be included in this chapter. The first is very popular for manufacture of smaller machines and very often there is a linear cylinder attached directly to the moving component.

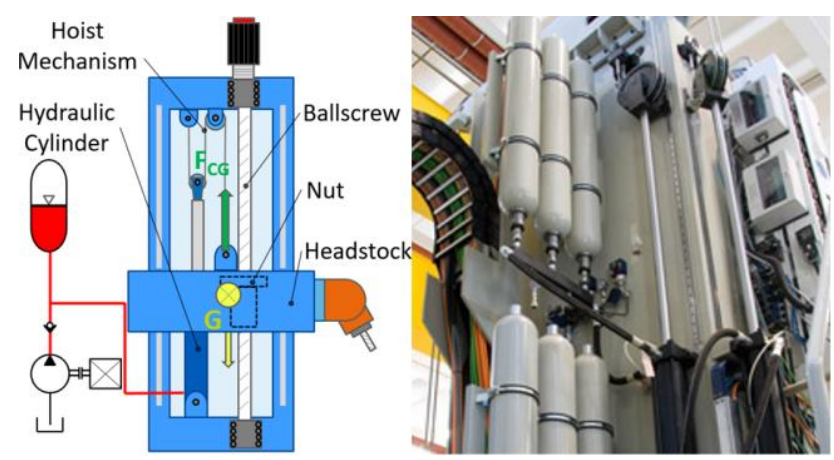

Fig. 5: Counterbalancing using hydraulic cylinders [Novotný, L. 2014].

This solution is widespread among manufacturers, with both pneumatic and hydraulic versions. The advantages are obvious: simple construction, reliability, durability (if properly mounted) and low costs. On the other hand, it is necessary to keep in mind that the cylinder has to be more than twice as long as the motion axis stroke. This requirement very often leads to use of very long slender cylinders which protrude from the machine.

To avoid this, a steel cable or multirow chain and pulleys or sprockets may be used to make a hydraulic hoist (Fig. 5). One of the most commonly stated disadvantages is similar to that of mechanisms with a counterweight and steel cable - specifically, the problem of oscillation of the large mass on the steel cable. Another disadvantage is that pulleys or sprockets are mounted on the top of the column. Sometimes these pulleys are mounted on a boom to move the point of the counterbalancing force and thus to compensate the loss due to ram extrusion. A large force situated on the top of the column can cause it to bend.

A rarely used technique is the usage of synchronized telescopic multisectional cylinders [Mittmann, H. 2013]. To avoid any impact to the superstructure of the machine, simultaneous movement of all sections of the cylinder must be ensured. This solution is very expensive and each synchronized cylinder is made to fit the specific machine.

To produce a constant force in a linear direction a rotary piston hydromotor with a mechanism which converts rotation to translation may be used. The hydromotor can be situated on the top of the column and equipped with a steel cable winding drum or it can be situated on a ballscrew as with the hydromotor variation. Another solution is to use two different mechanisms to convert rotation to translation - a gear rack and ballscrew. A concept which uses just a gear rack and gear pinion was developed at RCMT. The servo drive consists of a planetary gearbox; tooth belt transmission and gear pinion (Fig. 6).

This enabled the addition of an extra source of counterbalancing force by simply connecting the hydromotor directly to the gear pinion [Hovorka, J. 2015].

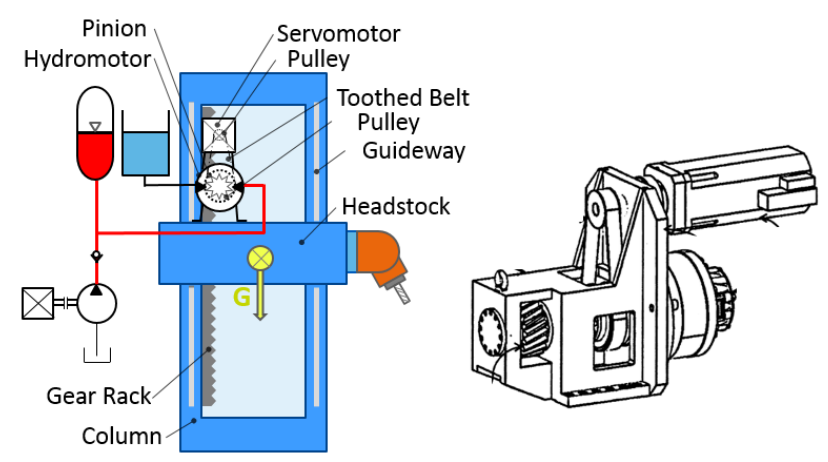

Fig. 6: Rotary piston hydromotor counterbalancing drive assembly [Hovorka, J. 2015].

One of the main intended advantages is that the height of the machine tool (axis stroke) is independent of the hydraulic mechanism. The only parameter associated with the axis stroke is the free volume of the nitrogen pressure cylinders.

\section{FIXED DISPLACEMENT ROTATIONAL HYDROMOTOR COUNTERBALANCING}

Usage of a rotational piston hydromotor is one of the fluid counterbalancing mechanisms. Using a rotational hydromotor as a source of torque is a very popular practice in the machine tool industry. Many leading manufacturers produce radial piston or axial piston hydromotors for this purpose. Hydromotor producers declare torque from very low speed, thus it appeared a good idea to use it as a source of constant torque for balancing. The main idea of using a rotational hydromotor is that this motor is directly mounted on the moving component and with use of the pinion, the whole assembly climbs the rack which is fixed to the column. The torque produced to compensate the gravitational force is led through the headstock and directly to the column via guideways where the force flow is closed. An ideal hydromotor would produce a constant torque and the whole drive works as shown in Fig. 7. A servomotor with a ballscrew is used for precise positioning and dynamic movements and the hydromotor with the pinion and rack provide only a counterbalancing function compensating the gravitational force.

Chapter 3.1 is dedicated to the experiment conducted on a machine tool, which revealed inconsistent behavior of the fixed displacement hydromotor. This undesirable behavior was confirmed by the experiment, which consisted of two motors (a hydromotor and servomotor) coupled directly together (Chapter 3.2).

A possible solution is described in Chapter 4, where the need to control the hydromotor is presented by the results on the experimental rig with the fixed displacement hydromotor (Chapter 4.1). Insufficient dynamic parameters achieved in the velocity and position closed loops led to a solution with torque control of the hydromotor, described in Chapter 4.2, which was fully functional.

\subsection{Machine Tool Experiment}

A completely assembled machine tool was equipped with a counterbalancing mechanism with a rotary hydromotor as shown in Fig. 7. The hydraulic circuit was checked and pressurized. Representative movement trajectories were established; in this case, the sinus line on the vertical axis simulates the circular interpolation of the end point of the tool. The resultant current chart is shown in Fig. 8, which clearly shows what happened when the axis reversed. 


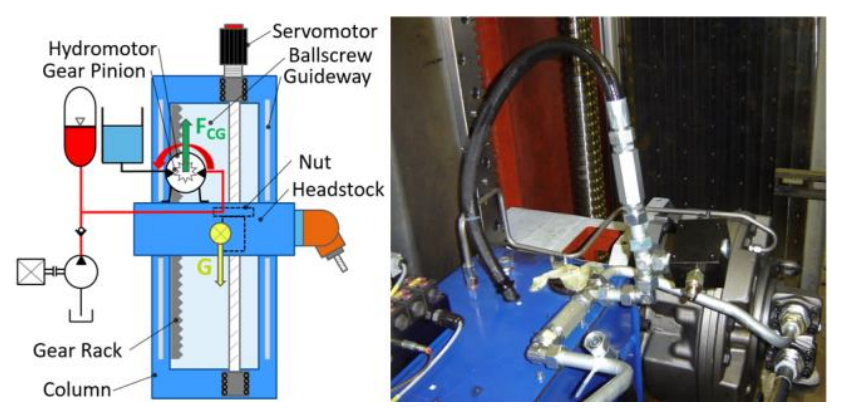

Fig. 7: Counterbalancing using a radial piston hydromotor and pinion and rack.

During the upwards motion, the current stays near zero. The current increased rapidly only when the axis changed direction (the hydromotor operation mode changed from motor to generator). This means that the hydromotor began to produce much more force during the downwards motion. This finally led to tilting the whole headstock due to the eccentric situation of both drives. Between forces, points of the lead screw and the pinion appeared to tilt momentarily, which disrupted the internal force balance. Another inconvenience was the periodic torque fluctuations. The weight of the headstock was 12 tons.
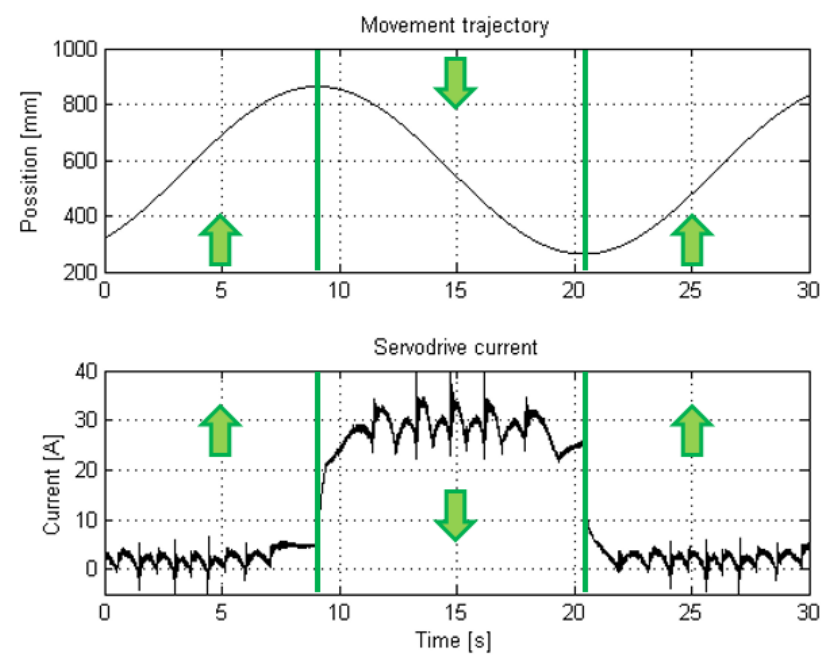

Fig. 8: Position and current record of the vertical feed drive with directional marks.

\section{Summary}

The headstock tilted with minute movements on the vertical axis, and the smoothness of movement was affected by large hydromotor torque fluctuations.

\subsection{Hydromotor Low Speed Forced Rotation}

Previous measurements had revealed some kind of problematic hydromotor behavior. The experimental rig was designed in order to understand and identify this behavior. The main goal of the experiment was to reveal whether the changing direction of rotation causes the high torque difference, how the hydromotor behaves at low speeds and how the fluctuation of torque affects the smoothness of the actual velocity of the feed drive.

During the first tests, all of the components were coaxially connected through a rigid steel frame. The whole assembly consists of an electric servomotor, planetary gearbox, flexible shaft coupling and radial piston hydromotor (Fig. 9). As you can see in Fig. 10 the hydraulic circuit was equipped with several pressure relief valves and a pressure reduction valve.

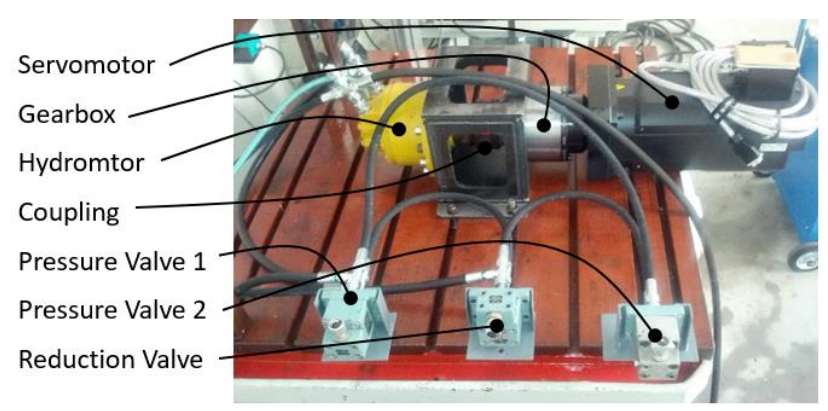

Fig. 9: Experimental rig for low RPM experiments.

This was necessary to ensure constant pressure on the return line. The force constant of the hydromotor was $8.9 \mathrm{Nm} / \mathrm{bar}$ and the frequency converter $32 \mathrm{~A}$. The torque constant of the servomotor was $2.876 \mathrm{Nm} / \mathrm{A}$ so the maximum torque $M_{\max }$, which the servomotor with planetary gearbox (7:1) could handle was:

$$
M_{\text {max }}=i k_{f} I_{\text {max }}=7 \times 2.876 \times 32=644.2[\mathrm{Nm}]
$$

where $i$ represents the gear ratio of the planetary gearbox, $k_{f}$ represents the force constant of the servomotor and $I_{\max }$ represents the maximal frequency converter current.

This condition 2) limited the maximal pressure drop $\Delta p_{\max }$ on the hydromotor:

$$
\Delta p_{\max }=\frac{M_{\text {max }}}{k_{f p}}=\frac{644.2}{8.9}=72.4[\text { bar }]
$$

where $k_{f p}$ represents the force constant of the hydromotor.

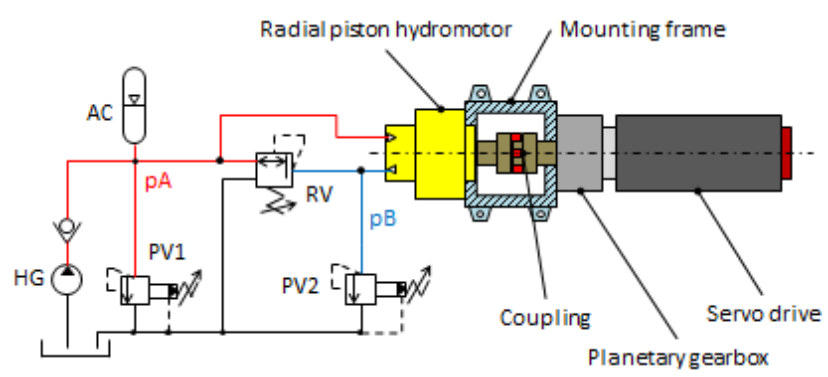

Fig. 10: Experimental rig for low rotational speed test (HG - hydrogenerator, AC - accumulator, PV - pressure relief valve, $R V$ - reduction valve).

The experiment consisted of several pressure sets and different rotational speeds. Perhaps the most telling chart is the one in Fig. 12. The first chart from the top shows two steps of velocity: the first is in the pressure drop direction and the second in the counter pressure drop direction.

This fully corresponds with current recordings (second chart from the top, Fig. 12). The current decreased while the motor was rotating in the pressure drop direction and significantly increased in the other direction. This could be caused by the hydromotor's operating mode:

\section{- Hydromotor}

\section{- Hydrogenerator}

In hydromotor mode the pressure in the inlet line (pA, Fig. 10, Fig. 12) could decrease and in hydrogenerator mode it could increase slightly, but this behavior is not observable on the third chart from the top - pressure recording. The pressure indication corresponds to both Fig. 10 and Fig. 12. The difference in current was measured in laboratory conditions (Fig. 11) and is associated with hydromotor behavior ( $\pm 6 \mathrm{~A}$ - converted to hydromotor corresponds to $\pm 120 \mathrm{Nm})$. 


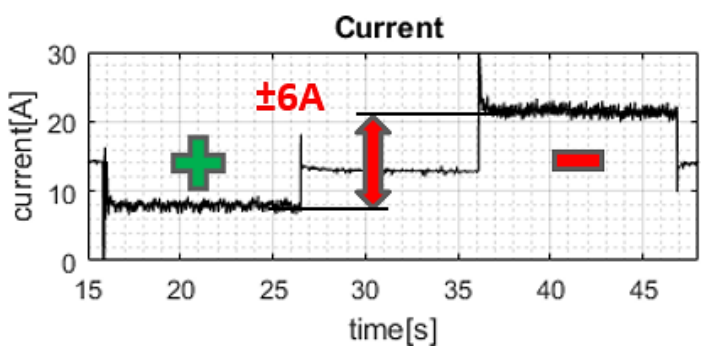

Fig. 11: Detail of current recording (+ pressure drop direction, - counter pressure drop direction).

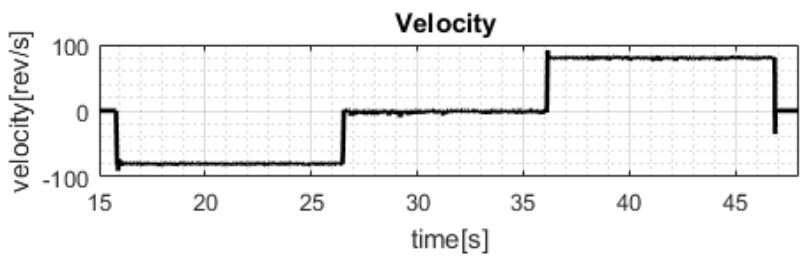

Current
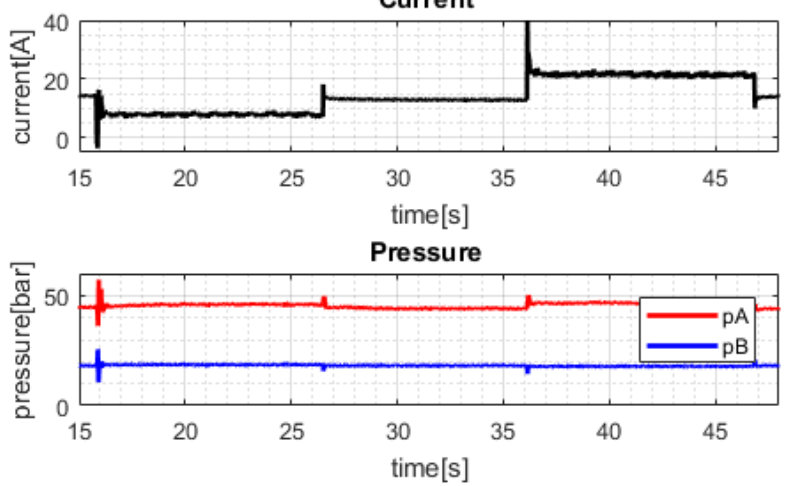

Fig. 12: Rotational speed, current and pressure chart.

\section{Summary}

The experiment confirmed the assumption that there is a significant difference in torque depending on the hydromotor/hydrogenerator mode change. This is a feature that is not desirable when used on machine tools. One possible solution lies in design, where the hydromotor is connected directly with the servomotor. Another solution is to use a variable displacement hydromotor and control it to suppress this undesirable behaviour.

\section{COMPACT COUNTERBALANCING UNIT WITH ROTATIONAL HYDROMOTOR}

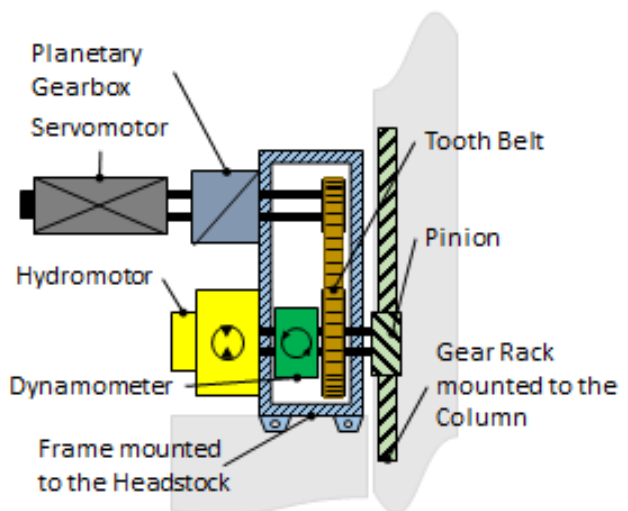

Fig. 13: Servo drive unit adapted with a counterbalancing hydromotor.
The measurements showed that the behavior of the rotational hydromotor was unsatisfactory and in response the counterbalancing mechanism was redesigned. The basic concept of the servo drive consists of the servomotor, planetary gearbox, tooth belt and gear pinion. Machine tool producers are very familiar with this concept. The only modification was coaxially connecting the hydromotor to the pinion drive shaft (see Fig. 13 and Fig. 14). Thus, the problematic behaviour of the hydromotor described in previous chapters could be captured directly by the servomotor drive. A torque transducer (dynamometer) was added to obtain relevant data for laboratory purposes.

\subsection{Fixed Displacement Hydromotor Experiments}

The main benefit of the compact servodrive unit with a counterbalancing hydromotor is that an ordinary, low-cost fixed displacement piston hydromotor is used for this purpose. Another big advantage is that the balancing mechanism is not dependent on the column design, especially not on its height. This construction enables the vertical moving axis to be designed with any stroke assuming suitable dimensioning of the hydraulic accumulators.

As shown in Fig. 15 the entire load is held by the electric servomotor during the non-counterbalanced state, which is represented by the black graph. During the upwards motion, torque reached approximately $220 \mathrm{Nm}$ and during the downwards motion the current decreased to $350 \mathrm{Nm}$. The servomotor was operated in standard positioning mode via EtherCAT with use of NI SoftMotion module.
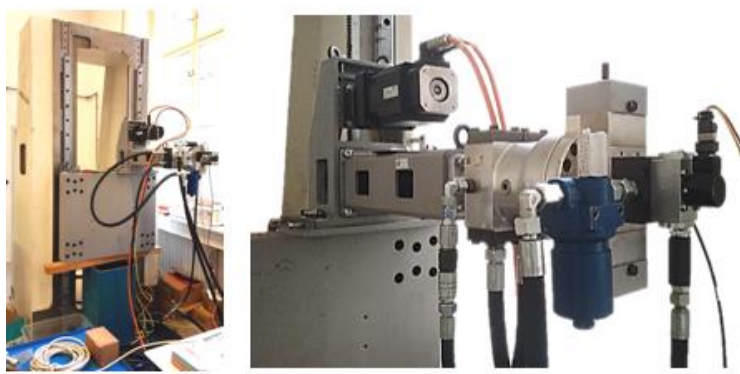

Fig. 14: Experimental rig for balancing vertical moving axes.

A decrease in torque is observable in the red graph, which represents the balanced state. During the downwards motion, torque reached $200 \mathrm{Nm}$ and during the upwards motion it dropped below zero to $-180 \mathrm{Nm}$. This means the servomotor pushes against the hydromotor, which is not required behavior. During both movements, torque should stay negative - one directional load.

Looking more closely at the required and actual positions reveals an issue associated with the stick-slip effect. As visible in Fig. 16, the downwards motion was worse and the velocity approached zero. The deviations varied in tenths of a millimeter. The solution with point of force situated into one point revealed another issue: torque could vary during hydro generator and hydromotor mode changing. Large passive resistance forces in the hydromotor caused vibrating movements near zero velocity. These conditions exclude this balancing method (fixed displacement hydromotor) from use on machine tools. Less demanding usage is definitely possible. 


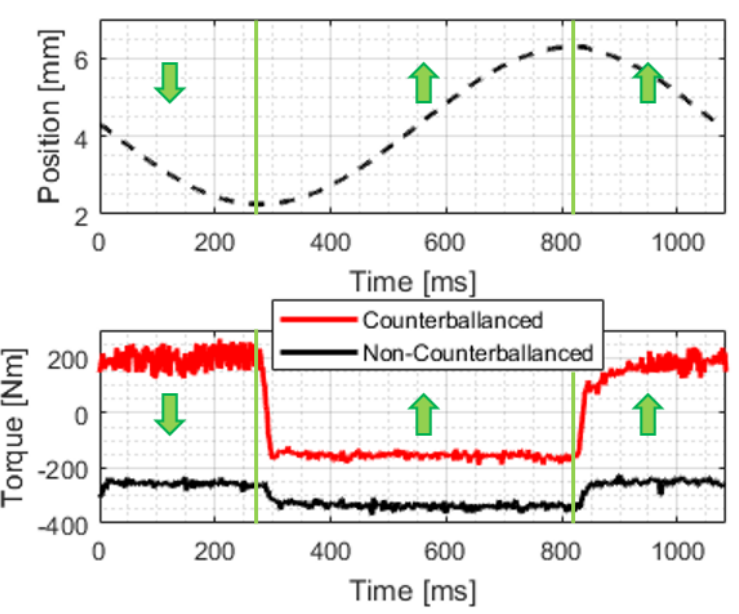

Fig. 15: Data measured during headstock movement.

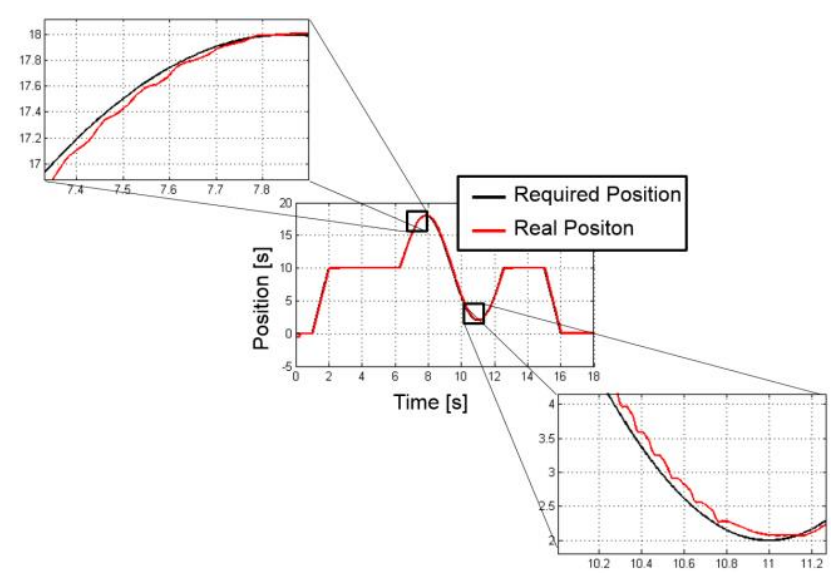

Fig. 16: Sine wave trajectory with stick-slip effect.

\subsection{Torque Control Counterbalancing Mechanism}

In order to ensure smooth vertical motion in both directions the axial piston hydromotor had to be regulated. There was a big difference in the torque of the hydromotor in the positive and negative directions that led to the closing of the force loop. The swivel plate of the axial piston hydromotor is activated by a servomechanism consisting of the servo valve, double rod cylinder and distance gauge. Thus it was possible to adjust the swivel plate angle precisely, thereby adjusting the hydromotor displacement.

\section{Closed Loop Hydromotor Control}

The variable displacement hydromotor could be controlled in a very similar way to the electric servomotor. Assuming that the pressure drop is constant or changes only negligibly, it is possible to imagine that output torque is dependent only on variable displacement [Pettersson, $\mathrm{K}$. 2008]. Thus variable displacement as a function of the swivel plate angle becomes analogous to the current in servodrive control attitude. The cascade servodrive control scheme consists of the current (force) loop, velocity loop and position loop [Souček, P. 2004]. The complete regulator scheme was adopted with only a few modifications. Current feedback is represented by hydromotor torque, so that it was necessary to convert the swivel plate position via volume displacement to torque (Fig. 18). By neglecting any offset between the swash plate and piston axis, the conversion of the displacement volume dependent on swash plate position may be deduced, i.e. sensed with an induction distance sensor. Fig. 17 shows that 3 pistons (out of 7) are always under pressure. Thus, the equivalent piston position and hydromotor displacement may be calculated, which results in the torque conversion formula:

$$
T_{q}(x)=S_{P} \Delta p \sum_{n} \frac{R}{r_{A}} x \sin \left(\varphi+\gamma_{n}\right)
$$

Where $T_{q}$ represents output hydromotor torque, $x$ represents swivel plate cylinder position, $x_{p 1,2,3}$ coordinates of 3 pistons under pressure, $r_{A}$ swivel axis and cylinder distance, $R$ piston drum radius, $\varphi$ rotation of main shaft, $\gamma$ piston phase, $S_{P}$ piston area and $\Delta p$ pressure drop.

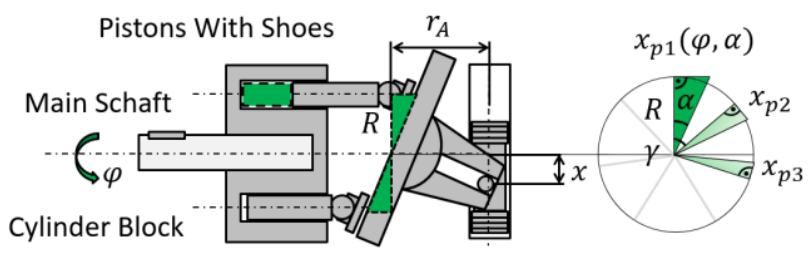

Fig. 17: Swash Plate Scotch Yoke Mechanism.

A simpler approach is linear hydromotor conversion:

$$
T_{q}(x)=\operatorname{sgn}(x) \frac{x}{x_{\max }} V_{g \max } \Delta p
$$

Where $x_{\max }$ is the maximum piston stroke position and $V_{\text {gmax }}$.

Both conversions are comparable, except for the neglected piston shoe offset error.

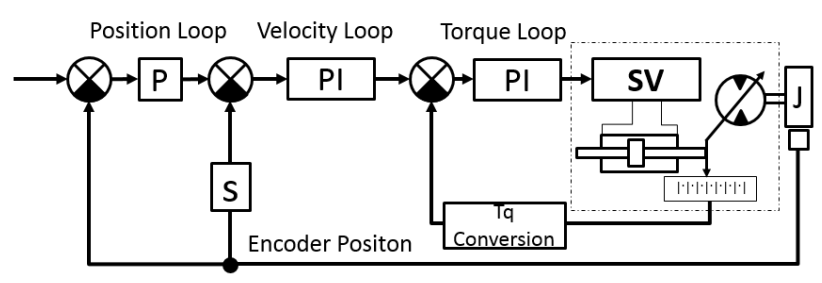

Fig. 18: Cascade regulator scheme (SV-Servo Valve, JInertia).

The bandwidth of the primary servo hydraulic displacement adjustment mechanism reaches about $95 \mathrm{~Hz}$. This is the most subordinate regulator loop, which should be the most quickly closed loop. The bandwidth of the other superior loops (velocity and position) are one tenth and one hundredth of $95 \mathrm{~Hz}$ frequency, respectively.

As mentioned above the dynamic behavior of the servo hydraulic drive is not sufficient to be coupled with an electric servo drive with use of a Master-Slave or Gantry regulatory scheme. Thus the main aim of the concept was to enable interaction between the electric servo drive and hydraulic servo drive in force loop to compensate the gravitational forces on the moving components.

\section{Compensating the Gravitational Force with Use of a Controlled Axial Piston Hydromotor}

The idea is based on a combination of a hydraulic servo drive, which is controlled in the force loop to compensate the gravitational force, and a purely hydraulic approach with basic energy accumulation in accumulators. In fact, another servo drive (hydraulic) is used to produce the counterbalancing force. Control scheme of the hydraulic servo drive is to see in Fig. 19.

The overall assembly of the servo drive unit is identical to that shown in Fig. 13, only with a variable displacement axial piston hydromotor instead of a fixed displacement hydromotor. This led to the possibility of controlling counterbalancing torque independently of the electric servo 
drive, which is used to accelerate and decelerate the axis and precise positioning.

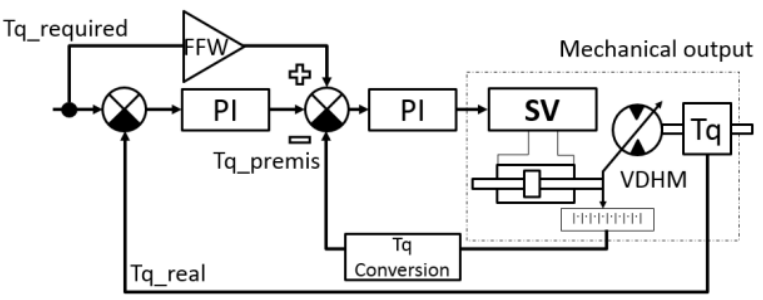

Fig. 19: Regulator scheme (FFW-Feedforward weight, SVServo Valve, Tq-Torque Transducer, VDHM-Variable Displacement Hydromotor).

Fig. 20 shows the current graph for counterbalanced and non-counterbalanced states. It is clear that the mean value for the upwards motion (see the trajectory in Fig. 20) decreases from $3.2 A$ to $0.1 A$ and for the downwards motion from $2 A$ to $0 A$. The entire mechanism is completely counterbalanced. A middle area $(3,500 \mathrm{~ms}-4,800 \mathrm{~ms})$ is observable where the required counterbalancing torque changed fluidly from a value corresponding to the upwards motion to the downwards motion.
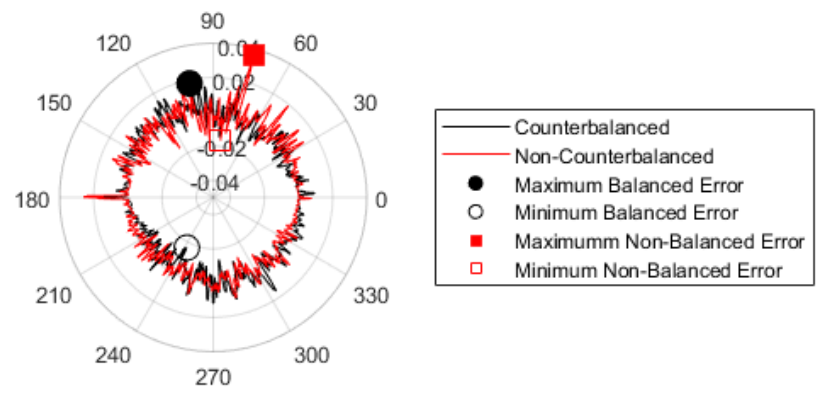

Fig. 21: Circularity test with $X$ axis simulation (units in polar graph $\mathrm{mm}$ ).

Another test that is analogous to tests performed during tuning of machine tools is a circularity test performed directly on control syste or by use of special hardware such as ballbar. Fig. 21 shows that with respect to accuracy, the counterbalanced state is comparable to the noncounterbalanced state. The horizontal axis is not present on the machine. For this reason the $\mathrm{X}$ axis of the polar chart is completely simulated (a smooth sinus wave with adequate frequency and amplitude). Thus it was possible to devise a circularity experiment with only one vertical moving axis.

Tab. 1: Comparison of counterbalanced and non-counterbalanced behavior during circularity test.

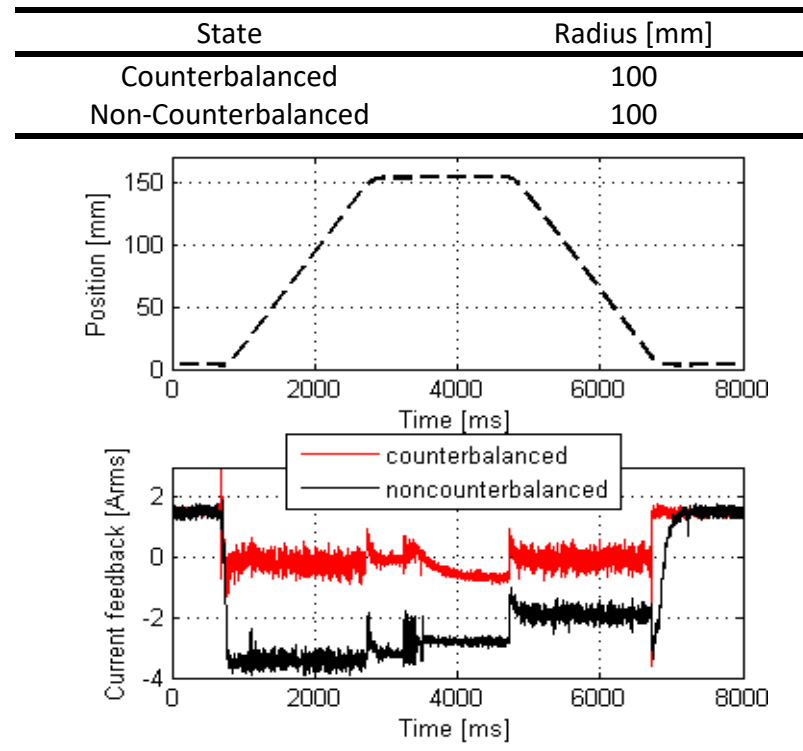

Fig. 20: Counterbalanced and non-counterbalanced comparison of current with compensation of directionaffected torque.

This example shows the mechanism's ability to include the influence of friction in the regulation of the counterbalancing mechanism to further reduce the load on the electric servo drive. The experimental rig for testing counterbalancing servo drive unit consists of just one gear rack and gear pinion. There is no other servo drive which would preload the gear pinion to compensate any backlash in gears. The only force which acts as a preloading factor is gravitational force itself. It should be noted that real machine tools are equipped with a mechanism which compensates backlash in gears. For such large machines, machine tool producers use two (one gear rack) or four (two gear racks) identical servo drive units. From the experimental point of view, the values reached during the experiments are adequate to the drive assembly used.

\begin{tabular}{cc} 
Maximal Error [mm] & Minimal Error [mm] \\
\hline 0.01775 & -0.01685 \\
0.03641 & -0.01702 \\
\hline
\end{tabular}

Comparison of position errors is in Tab. 1. As can be seen most of the inequalities are naturally situated on the top and bottom exact centers. This test expresses the relative comparison of the two states of the machine at the dead center and the comparison of the impact of the stick slip effect. It is obvious that with help of hydraulic servo drive it is possible to cope with friction forces and keep position error in reasonable values.

\section{DISCUSSION}

The solution with a rotational hydromotor introduces the possibility of using it in machines with any strokes of vertical axis. The only factor which depends on the stroke is hydraulic accumulator sizing. However, there are some issues such as torque fluctuation, stick-slip effect and the large torque difference in hydromotor/hydrogenerator mode changes. The inconveniences for use in manipulation and transport equipment are negligible. These issues must be addressed for application of counterbalancing with a rotary piston hydromotor on machine tools.

This inconvenient behavior of the fixed displacement hydraulic motor was suppressed by controlling the hydromotor in the feedback loop. Such a servo drive unit consists of a standard electric servo drive equipped with a planetary gear box and final toothed belt drive. There is the hydraulic servo mechanism in parallel, which tracks the movement and provides support for the electric drive by adding a counterbalancing force. In comparison to pure electric variation, there is no significant deterioration of the quality parameters.

\section{SUMMARY}

The paper describes new knowledge in hydromotor regulation in accordance with an electric servo drive and its influence. Simulations and practical experiments were used to prove that it is possible to use a rotational hydromotor, 
as a part of a counterbalancing mechanism, only in controlled service. The designed control approach, despite the inconvenient behavior of the hydraulic servo drive, which is associated with the properties of the specific hydromotor, is able to counterbalance the vertical moving axis of the machine tool. Stable and smooth control of the hydromotor resulted in good electric servomotor control quality (smoothness, current scatter, position error). Through experimentation it was possible to reduce the constant current flow through the electric servo drive from $3 A$ to $-0.3 A$ (mean values, Fig. 20). The advantage of being able to design any machine tool height without needing to consider hydraulic or pneumatic cylinder length is obvious. The same counterbalancing unit may be used for the entire range of the machine tool, which adopts similar headstocks.

\section{ACKNOWLEDGMENTS}

The presented results were achieved within the project TE01020075 funded by Technology Agency of Czech Republic.

\section{REFERENCES}

\section{Papers in journals:}

[Kolář, P. 2017] Kolář, P., Smolík, J., Sulitka, M., Šindler, J., and Hovorka, J. An Integrated Approach to the Development of Machine Tool Structural Parts, MATAR2012-12082, MM Science Journal, Prague.

[Lin, S. 2008] Lin, S., Y., Fang, Y. C., and Huang, C. W. Improvement strategy for machine tool vibration induced from the movement of a counterweight during machining process, International Journal of Machine Tools and Manufacture, Vol. 48, No.7, pp. 870-877.

[Möhring, H.-C. 2015] Möhring, H.-C., Brecher, C., Abele, E., Fleischer , J., and Bleicher ,F. Materials in machine tool structures, CIRP Annals - Manufacturing Technology, Vol. 64, No.2, pp. 725-748.

[Novotny, L. 2014] EMO Hannover 2013: part 7 Technologies that are not often seen, <http://www.mmspektrum.com/clanek/emo-hannover-

2013-cast-7-technologie-ktere-mnohdy-nejsou-videt.html> (Apr. 20, 2017).

[Zhao, L. 2011] Zhao, L., Ma, J., Chen, W., and Guo, H. Lightweight Design and Verification of Gantry Machining Center Crossbeam Based on Structural Bionics, Journal of Bionic Engineering, Vol. 8, No.2, pp. 201-206.

\section{Technical reports and theses:}

[Fermat 2017] [n.d.]
http://www.fermatmachinery.com/wrf130-cz (May 2, 2017).
http://www.fermatmachinery.com/wrf130-cz (May 2, 2017).

[Hovorka, J. 2015] Compact driving unit with balancing vertical linear moving axes of machine tools.

[ICON Technologies Home of the ICON 2017] [n.d.] http://icon.hydromat.com/tripod.html (May 4, 2017).

[Loxin Industrial Applications 2017] [n.d.] http://www.loxin2002.com/movable-structure-gantryconfiguration (Apr. 25, 2017).

[Mittmann, H. 2013] Hydraulischer Gleichlaufteleskopzylinder.

[Munirathnam, M. 2008] Einfluss masseoptimierter Kragarmstrukturen auf die dynamische Bahngenauigkeit von HSC-Fräsmaschinen, Innovation Fertigungstechnik.

[Pettersson, K. 2008] Secondary Controlled Swing Drive.

[ŠKODA MACHINE TOOL. 2017] [n.d.] http://www.skodamt.com/-1/ (May 4, 2017).
[Souček, P. 2004] Servomechanisms In Production Machines, ČVUT.

[Turning: Emco Group 2015] [n.d.] http://www.emcoworld.com/en/products/industry/turning/cat/90/d/2/p/10002 96,90/pr/emco-vertical-turn-vt-250/view/6.html (Jul. 1, 2015) 